exacerbation HDAC2 activity fell in COPD subjects, this represents a potential mechanism of excessive inflammation. The same pattern is not seen in control subjects.

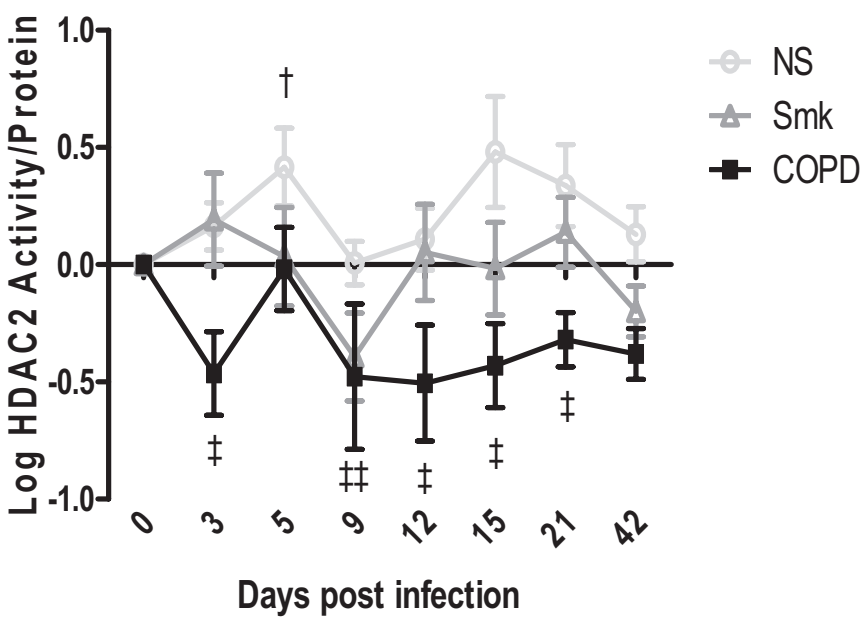

Abstract S14 figure 1 Time course of sputum macrophage HDAC2 activity in individual groups normalised for baseline. Data presented mean \pm SEM. (Paired t test between day 0 and post infection day $\dagger$ in NS $p<0.05 ; \ddagger$ in COPD $p<0.05$, $\ddagger$ in COPD $p<0.01$ ).

\section{S15 DETECTION OF BACTERIA IN SPUTUM FOLLOWING EXPERIMENTAL RHINOVIRUS INFECTION IS MORE COMMON IN COPD THAN CONTROLS SUBJECTS}

doi:10.1136/thoraxjnl-2011-201054b.15

J I M Footitt, P Mallia, M B Trujillo-Torralbo, A Jepson, S L Johnston. NHLI, Imperial College, London, UK

Introduction and Objectives There is increasing evidence that the majority of acute exacerbations of COPD (AECOPD) are caused by virus infection, and rhinoviruses are the most frequently identified species. Bacteria are also responsible for AECOPD but the relationship between these two is poorly understood. To investigate this further bacterial culture was performed in sputum samples collected from GOLD stage II COPD subjects $(n=9)$ and non-obstructed smoking $(\mathrm{n}=10)$ and non-smoking $(\mathrm{n}=11)$ controls enrolled in a rhinovirus challenge study.

Methods Rhinovirus infection was confirmed with quantitative PCR performed on nasal lavage and sputum samples collected at baseline and days $3,5,9,12,15,21$ and 42 post virus inoculation. Semi-quantitative bacterial detection was performed in sputum samples by a CPA-accredited microbiological laboratory. Any subject that had bacteria detected on or after day 9 was defined as "bacteria positive" and those with none detected were defined "bacteria negative". Species frequently associated with respiratory illness were defined as pathogenic ( $S$ pneumoniae, $H$ influenzae, $M$ catarrhalis and $S$ aureus) and any others as non-pathogenic.

Results No bacteria were detected in baseline sputum samples. Peak virus load occurred on day 9 with maximum bacterial colonies identified on day 15 . There were significantly more bacteria positive subjects in the COPD group (67\%) with the majority of control subjects (81\%) being classified as bacteria negative, (Abstract S15 figure $\left.1, \chi^{2} \mathrm{p}=0.04\right)$. COPD subjects with bacteria detected at any time point in the study had significantly more pathogenic species in their sputum samples $(n=8 / 8)$ compared to controls $(n=1 / 9),\left(\chi^{2}\right.$ $\mathrm{p}=0.0001)$. No non-pathogenic bacteria were detected in COPD subjects.

Conclusions Detection of bacteria is common after rhinovirus infection, with the peak occurring 6 days after maximum virus load.
COPD subjects are more likely to have pathogenic bacteria detected than controls following virus infection. Mechanisms responsible for this phenomenon merit further investigation.

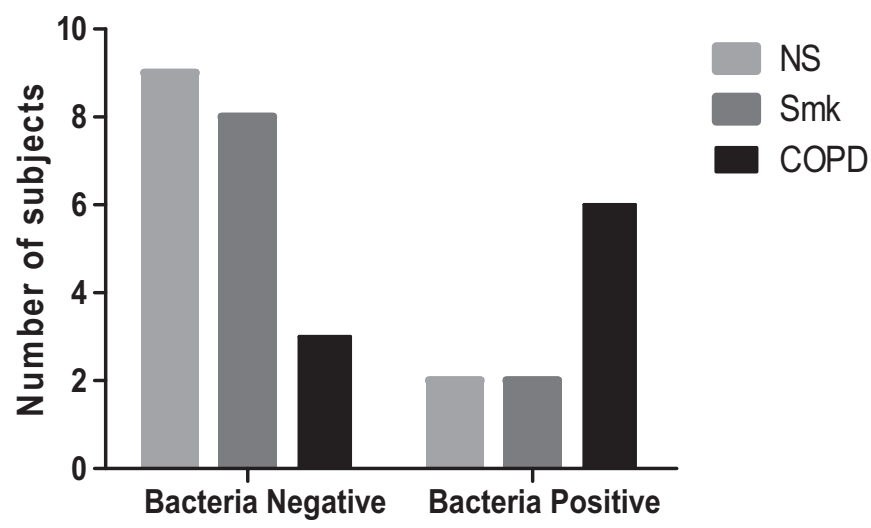

Abstract S15 Figure 1 Number of subjects in each study group with bacteria negative or bacteria positive sputum samples on or following day $9,\left(\chi^{2} p=0.04\right)$.

\section{S16 MOLECULAR PROFILING OF THE AIRWAY MICROBIOME IN COPD}

doi:10.1136/thoraxjnl-2011-201054b.16

K Haldar, K Lau, M Bafadhel, C Brightling, M Barer. University of Leicester, Leicester, England

Introduction It is hypothesised that bacteria are important in the pathogenesis of COPD exacerbations and clinical expression of disease. To date, most bacteriological research in COPD has been performed using culture based methods. However, novel molecular approaches offer more detailed evaluation of the airway microbiome that may better inform the role of bacteria in COPD.

Aims To characterise the microbial community in COPD and examine whether detectable changes occur with serial longitudinal assessment at stable, exacerbation, follow-up and recovery visits.

Methods 115 COPD patients that were part of a clinical trial had sputum samples collected at the four time points. Patients received antibiotics and / or oral corticosteroids after clinical assessment to treat exacerbations. Follow-up and recovery samples were collected 2 and 6 weeks after the exacerbation sample. Real-time quantitative PCR (qPCR) was performed on sputum DNA using universal 16S gene primers and specific gene targets to quantify total bacterial load and the specific pathogens Haemophilus influenzae, Streptococcus pneumoniae, Moraxella catarrhalis and Staphylococcus aureus. In a subgroup of 30 patients, 454 high-throughput pyrosequencing was performed at each of the 4 visits to examine changes to the global microbiome.

Results Quantitative PCR identified one or more pathogens in $94 \%$ of stable samples and $97 \%$ of exacerbation samples. There was no significant difference in the total bacterial load or any specific pathogen between longitudinal stable and exacerbation samples. 454 pyrosequencing identified Proteobacteria and Firmicutes to be the dominant groups contributing $>80 \%$ of the sequence reads at phylum level. Haemophilus, Moraxella and Streptococcus were the dominant groups at genus level. Cluster analysis characterised three groups on the basis of the ratio of Proteobacteria to Firmicutes. No significant differences in patient characteristics were observed between microbiome clusters. There was no significant change across visits in the microbial community at either phylum or genus level. No treatment specific effects on the microbiome were observed. 